\title{
A CMB search for the neutrino mass mechanism and its relation to the Hubble tension
}

\author{
Miguel Escudero ${ }^{1, \mathrm{a}}{ }_{\mathbb{1}}$, Samuel J. Witte $^{2, \mathrm{~b}}{ }_{(\mathbb{C})}$ \\ ${ }^{1}$ Department of Physics, King's College London, Strand, London WC2R 2LS, UK \\ ${ }^{2}$ Instituto de Física Corpuscular (IFIC), CSIC-Universitat de València, València, Spain
}

Received: 13 January 2020 / Accepted: 17 March 2020 / Published online: 1 April 2020

(C) The Author(s) 2020

\begin{abstract}
The majoron, a pseudo-Goldstone boson arising from the spontaneous breaking of global lepton number, is a generic feature of many models intended to explain the origin of the small neutrino masses. In this work, we investigate potential imprints in the cosmic microwave background (CMB) arising from massive majorons, should they thermalize with neutrinos after Big Bang Nucleosynthesis via inverse neutrino decays. We show that $\mathrm{Planck} 2018$ measurements of the CMB are currently sensitive to neutrino-majoron couplings as small as $\lambda \sim 10^{-13}$, which if interpreted in the context of the type-I seesaw mechanism correspond to a lepton number symmetry breaking scale $v_{L} \sim \mathcal{O}(100) \mathrm{GeV}$. Additionally, we identify parameter space for which the majoronneutrino interactions, collectively with an extra contribution to the effective number of relativistic species $N_{\text {eff }}$, can ameliorate the outstanding $H_{0}$ tension.
\end{abstract}

\section{Introduction}

Despite unambiguous evidence that at least two of the known neutrinos have a non-zero mass, the Standard Model (SM) is still lacking of an explanation of their origin. Perhaps more concerning, however, is the question of why neutrino masses are so much smaller than those of charged leptons. While many models have been proposed over the years to explain both the origin and smallness of the neutrino masses (see e.g. [1-6]), perhaps the most compelling class of models are those which invoke the so-called seesaw mechanism [711]. In such scenarios, the SM is augmented by heavy righthanded neutrinos carrying a Majorana mass term $m_{N}$, which

Electronic supplementary material The online version of this article (https://doi.org/10.1140/epjc/s10052-020-7854-5) contains supplementary material, which is available to authorized users.

a e-mail: miguel.escudero@kcl.ac.uk (corresponding author)

be-mail: sam.witte@ific.uv.es naturally give rise to light neutrino masses $m_{v}$ of the order $\sim y_{N}^{2} v_{H}^{2} / m_{N}$, where $v_{H} \simeq 246 \mathrm{GeV}$ is the vacuum expectation value of the SM Higgs, and $y_{N}$ is the Dirac Yukawa coupling of the right-handed neutrinos. Generating the Majorana mass term necessary to implement the seesaw mechanism is often accomplished by introducing a new scalar that spontaneously breaks lepton number. Assuming that lepton number is a global symmetry, as in the SM, the spontaneous symmetry breaking (SSB) triggered by the scalar leads to the prediction of a pseudo-Goldstone boson, the so-called majoron [12] (see also [13-15]).

The majoron is notoriously difficult to probe since it interacts very weakly with all SM particles, particularly with charged fermions $\lambda_{\phi e} \sim 10^{-20}$ [12]. However, measurements of the Cosmic Microwave Background (CMB) have reached a level of precision where small modifications to the neutrino sector may be discernible [16-34]. The effect of including majoron-neutrino interactions in the early Universe are twofold [17]: (i) they lead to a non-standard expansion history after Big Bang Nucleosynthesis $(\mathrm{BBN})$ and prior to recombination (generically amounting to $\Delta N_{\text {eff }} \sim \mathcal{O}(0.1)$ ), and (ii) they act to suppress the neutrino anisotropic stress energy tensor, and hence reduce neutrino free-streaming [16]. The idea of identifying features in the CMB arising from the majoron, and thus providing an indirect probe of the neutrino mass mechanism, was proposed at the start of the century [17]. However, until now, no rigorous cosmological implementation of this idea has been performed, ${ }^{1}$ nor has there been an analysis using real data.

Using Planck2018 data [35,36], we analyze a wellmotivated region of parameter space in which majorons

\footnotetext{
${ }^{1}$ References [20-22] explored the possibility that some component of radiation contained strong self-interactions; this was accomplished by artificially setting to zero the multiples $\ell \geq 2$ in the Boltzmann hierarchy for the interacting radiation. This approach, however, cannot be applied (or mapped) into the scenario of [17], since neutrino-majoron interactions rates are strongly time-dependent and not infinite in strength.
} 
thermalize with neutrinos after BBN via inverse neutrino decay. We show that neutrino-majoron couplings as small as $10^{-13}$ can be robustly excluded with existing CMB data; future experiments, such as the Simons Observatory [37] and CMB-S4 [38], which are aiming to probe the effective number of relativistic species $N_{\text {eff }}$ at the sub-percent level, could have sensitivity to couplings as small as $10^{-14}$. If interpreted in the context of the type-I seesaw model, these couplings point toward a lepton number symmetry breaking scale of $\mathcal{O}(100) \mathrm{GeV}$ and $\mathcal{O}$ (1) $\mathrm{TeV}$, respectively. Thus, quite remarkably, the $\mathrm{CMB}$ is providing an indirect probe of the neutrino mass mechanism at collider energy scales (albeit unaccessible to colliders due to their small couplings), but using feeble interactions with neutrinos in the early Universe.

While the $\Lambda \mathrm{CDM}$ model has been incredibly successful at describing both high- and low-redshift cosmological observations, a concerning tension has recently emerged between the value of the Hubble constant $H_{0}$ inferred using early Universe observations (with data either from the CMB [35], or by combining measurements from BBN with baryonic acoustic oscillations, i.e. BAOs [39-41]), and various local late Universe measurements performed using observations of type-Ia supernovae (see e.g. [42-47]) and strong lensing [48-51] (see e.g. [52] for an overview of the various measurements). The most prolific of these discrepancies is between the value inferred by Planck, $H_{0}=67.4 \pm 0.5 \mathrm{~km} / \mathrm{s} / \mathrm{Mpc}$ [35], and that observed by $\mathrm{SH}_{0} \mathrm{ES}$ collaboration, which relies on cepheids to calibrate the distance to type-Ia SN, who find a value of $H_{0}=74.0 \pm 1.4 \mathrm{~km} / \mathrm{s} / \mathrm{Mpc}$ [43]. Depending both on the choice of distance calibration and how one chooses to combine datasets, the outstanding tension is determined to be at the level of $\sim 4-6 \sigma[52,53]$. While it is of course possible that this tension is a consequence of unaccounted for systematics in either or both measurements, throughout this work we will take this discrepancy at face value and assume alternatively that this is an indication of new physics beyond the $\Lambda$ CDM paradigm.

Various groups have attempted to resolve this issue by including additional contributions to $N_{\text {eff }}$ [54-58], strong neutrino self-interactions [30,31], hidden neutrino interactions [59-61], exotic dark energy models [62-75], dark sector interactions [76-79], and modified theories of gravity [8082]. Most of these solutions are either incapable of resolving the tension fully [83-85], are experimentally constrained [86], are highly fine-tuned, or lack theoretical motivation. Perhaps the most simple, and thus theoretically appealing, solution which can ameliorate the $H_{0}$ tension to the level of $\sim 3 \sigma$ is simply to postulate the existence of non-interacting dark radiation producing a shift in the radiation energy density relative to the value predicted in the Standard Model of $\Delta N_{\text {eff }} \sim 0.25$. A more appealing, albeit far more problematic, solution was introduced in [30], where it was shown that strongly interacting 2-to-2 neutrino scatterings together with a contribution to $\Delta N_{\text {eff }} \sim 1$ was able to fully resolve the tension; unfortunately, this solution requires neutrino couplings that are not phenomenologically viable [86], a value of $\Delta N_{\text {eff }}$ excluded by BBN [87], and is only successful at reducing the tension if $\mathrm{CMB}$ polarization data is neglected. Given that the majoron naturally contributes to $\Delta N_{\text {eff }}$ at the level of $\sim 0.11$ via late-time thermalization and decay, and damps neutrino free-streaming in a manner similar to that of the strongly interacting neutrino solution, it is natural to ask whether 2-to-1 neutrino-majoron interactions are capable of further reducing the $H_{0}$ tension, beyond what is simply accomplished with $\Lambda \mathrm{CDM}+\Delta N_{\text {eff }}$. Indeed we show that including majoron-neutrino interactions broadens the posterior such that the $H_{0}$ tension can be further reduced, albeit only to the level of $2.5 \sigma$, a level that is comparable with other viable solutions, such as early dark energy (see e.g. [70]).

\section{Majoron interactions}

We parametrize the majoron-neutrino interaction as:

$\mathcal{L}=i \frac{\lambda}{2} \phi \bar{v} \gamma_{5} \nu$

where $v$ corresponds to a light neutrino mass eigenstate. The coupling $\lambda$, taken here to be universal, is typically intimately related to the mass of the active neutrinos $m_{v}$ and the scale at which lepton number is spontaneously broken, $v_{L}$. For example, in the type-I seesaw mechanism, $\lambda$ can be expressed as

$\lambda=2 U^{2} \frac{m_{N}}{v_{L}} \simeq 2 \frac{m_{v}}{v_{L}}$,

where $U$ is the mixing between sterile and active neutrinos, and the last line follows from a condition in the type-I seesaw that $U^{2} \sim m_{v} / m_{N}$ [6]. Interestingly, for values of $v_{L} \sim v_{H}$ and neutrino masses consistent with current constraints, the value of $\lambda$ within this model can e.g. naturally be of the order of $\lesssim 10^{-12}$, which happens to be around the region where inverse neutrino decays $(\bar{v} v \rightarrow \phi)$ can thermalize light majorons after BBN, but prior to recombination. In what follows we will treat $\lambda$ as a free parameter to remain as model-independent as possible, and when appropriate, relate $v_{L}$ to $\lambda$ by considering the atmospheric mass splitting $m_{v} \sim \sqrt{\left|\Delta m_{\mathrm{atm}}^{2}\right|} \simeq 0.05 \mathrm{eV}$ [88]. Namely, $v_{L} \simeq 1 \mathrm{TeV}\left(10^{-13} / \lambda\right)$.

\section{The majoron mass}

Quantum gravity is expected to break all global symmetries [92,93], and hence the majoron should acquire a small but 


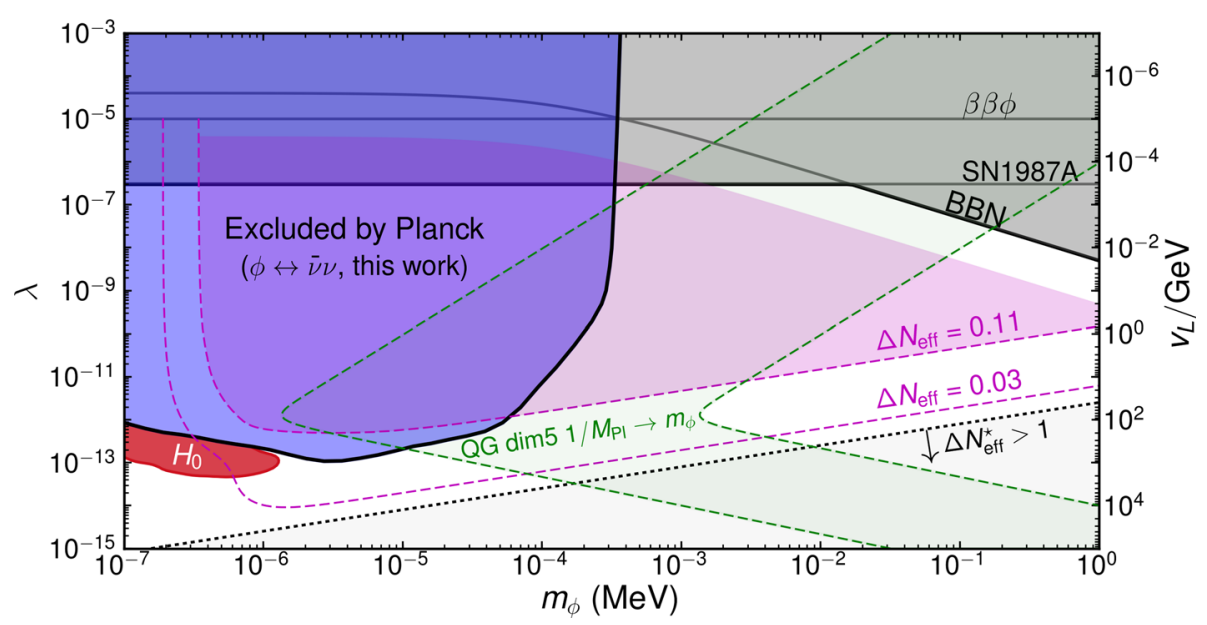

Fig. 1 Majoron parameter space. The left and right vertical axes correspond to the majoron-neutrino coupling and the scale at which lepton number is spontaneous broken in the type-I seesaw model respectively. Current constraints from KamLAND-Zen [89], BBN (see text), and SN1987A $[90,91]$ are shown in grey. The pink region demarcates parameter space for which the majoron fully thermalizes after neutrino decoupling, leading to $\Delta N_{\text {eff }}=0.11$. The green band highlights the

non-zero mass. Naively, one might expect the majoron mass to arise from dimension-five (dim-5) Planck scale suppressed operators $[94,95]$. Should these dim-5 operators involve only the Higgs and the scalar responsible for the SSB of lepton number, the majoron mass is expected to be

$m_{\phi}^{2} \sim \frac{\beta}{0.1} \frac{v_{H}}{v_{L}}\left[1+35\left(\frac{v_{L}}{v_{H}}\right)^{4}\right] \mathrm{keV}^{2}$

where $\beta$ is the coupling constant of a given operator at the Planck scale - which, for concreteness, we have assumed to be the same for all relevant dim-5 operators [95]. Of course, the actual details of the breaking of global symmetries by gravity depend upon the unknown quantum nature of the gravitational theory at the Planck scale [96]; thus we treat $m_{\phi}$ as a free parameter in this work, centered approximately around the $\mathrm{keV}$ scale, but allowed to vary from $0.1 \mathrm{eV}$ to $1 \mathrm{MeV}$.

Figure 1 contains a depiction of the majoron parameter space relevant for this work. In addition to highlighting parameter space currently excluded by SN1987A [90,91], BBN (see Supplementary Material, and e.g. [97]), and KamLAND-Zen [89], we identify masses and couplings for which the majoron is consistent with arising from dim-5 Planck scale suppressed operators. ${ }^{2}$ We defer discussion of the remainder of this plot to later sections.

\footnotetext{
2 This band is constructed by varying $\beta$ in Eq. (3) between $10^{-6}$ and 1 , where the lower/upper limit has been chosen to be reflect the electron/top Yukawa coupling.
}

region of parameter space in which the majoron mass could arise from dim-5 Planck suppressed operators (3). Shown in blue is the parameter space excluded in this work using Planck2 018 data at 95\% CL. The parameter space below the black dotted line is excluded if there was a small but primordial population of thermal majorons. The region labeled ' $H_{0}$ ' is the preferred $1 \sigma$ contour for resolving the Hubble tension

\section{Model extensions}

Looking forward, it may be interesting to consider the possibility that one of the active neutrinos is exactly massless, as this would decouple the lightest neutrino form the majoron, changing the cosmological evolution of the system. One could also conceive of the possibility of a multi-majoron system resulting from the SSB of a more complex flavor symmetry group in the neutrino sector [17]. In such a scenario, one could produce a more complicated thermalization history which produces step-like features in the evolution of the energy density, and damps the perturbations in a nontrivial manner. While these models are beyond the scope of the current work, they provide a clear extension of the ideas and prospects studied here.

\section{Early universe cosmology}

The collision terms governing the evolution of the neutrino and majoron phase space distributions are determined by the decay rate of the majoron into two neutrinos $\phi \rightarrow \bar{v} \nu$, given by

$\Gamma_{\phi}=\frac{\lambda^{2}}{16 \pi} m_{\phi} \sqrt{1-\frac{4 m_{v}^{2}}{m_{\phi}^{2}}} \simeq \frac{\lambda^{2}}{16 \pi} m_{\phi}$,

where in the last step we have considered $m_{v} \ll m_{\phi}$. In order to model the time-dependent evolution of the number density and energy density of the system, we follow $[98,99]$ in 
assuming that all relevant species are characterized by a temperature $T_{i}$ and chemical potential $\mu_{i}$, and solve for their time evolution accounting for all relevant interactions ${ }^{3}$ (see Supplementary Material for details). If the majoron is sufficiently heavy and interactions sufficiently strong, the majorons may begin to thermalize prior to or during $\mathrm{BBN}$, leading to an enhanced expansion history of the Universe that would alter the formation of the light elements. For small couplings and masses $\left(\lambda \lesssim 10^{-5}\right.$ and $\left.\lambda \lesssim 10^{-10} \mathrm{MeV} / m_{\phi}\right)$, majorons thermalize with neutrinos after BBN, and when the majorons become non-relativistic at $T_{v} \sim m_{\phi} / 3$, they decay out of equilibrium to neutrinos leading to a small enhancement in $N_{\text {eff }}$, which asymptotes to $\Delta N_{\text {eff }}=0.11$. We identify in Fig. 1 a shaded pink region for which full thermalization is achieved after BBN. For yet smaller couplings, partial thermalization can be achieved; the dashed pink line in Fig. 1 identifies majorons that never thermalize, but augment $N_{\text {eff }}$ to a level that may be observable with CMB-S4 experiments [100].

We model the phase space perturbations by considering the coupled neutrino-majoron fluid, and approximate the entire system as being massless. ${ }^{4}$ Despite the fact that the temperature of the Universe eventually becomes similar to the majoron mass, the majoron contribution to the energy density of the neutrino-majoron system is never larger than $10 \%$. We have explicitly verified that the equation of state $\omega=\left(p_{\phi}+p_{\nu}\right) /\left(\rho_{\phi}+\rho_{\nu}\right)$ and the speed of sound $c_{s}^{2}=\delta\left(p_{\phi}+p_{\nu}\right) / \delta\left(\rho_{\phi}+\rho_{\nu}\right)$ deviate by less than $3 \%$ with respect to that of an ultra-relativistic fluid, i.e. $\omega=c_{s}^{2}=1 / 3$ (see Supplementary Material). Additionally, we adopt the relaxation time approximation for the collision term [105], which has been shown to accurately reproduce the full solution in similar scenarios [26,27]. The above simplifications allow us to express the density contrast $\delta$, the fluid velocity $\theta$, the shear $\sigma$, and the higher anisotropic moments in the synchronous gauge as $[105,106]$ :

$$
\begin{aligned}
\dot{\delta}_{\nu \phi}= & -\frac{4}{3} \theta_{\nu \phi}-\frac{2}{3} \dot{h}, \\
\dot{\theta}_{\nu \phi}= & k^{2}\left(\frac{1}{4} \delta_{\nu \phi}-\sigma_{\nu \phi}\right), \\
\dot{F}_{\nu \phi 2}= & 2 \dot{\sigma}_{\nu \phi}=\frac{8}{15} \theta_{\nu \phi}-\frac{3}{5} k F_{\nu \phi 3} \\
& +\frac{4}{15} \dot{h}+\frac{8}{5} \dot{\eta}-2 a \Gamma \sigma_{\nu \phi},
\end{aligned}
$$

\footnotetext{
3 Reference [98] explicitly demonstrates that this method accurately reproduces a full numerical solution to the Liouville equation for the neutrino and majoron distribution functions within the relevant parameter space considered in this study.

4 The error introduced by neglecting neutrino masses is the Boltzmann hierarchy is expected to be entirely negligible given current constraints on $\sum m_{v}<0.12 \mathrm{eV}$ [35], see also [101-104].
}

$$
\begin{aligned}
\dot{F}_{\nu \phi \ell}= & \frac{k}{2 \ell+1}\left[\ell F_{\nu \phi(\ell-1)}-(\ell+1) F_{\nu \phi(\ell+1)}\right] \\
& -a \Gamma F_{\nu \phi \ell} \text { for } \ell \geq 3 .
\end{aligned}
$$

Here, $h$ and $\eta$ account for the metric perturbations, $k$ is a given Fourier mode, $F_{v \phi \ell}$ represents the $\ell^{\text {th }}$ multipole, $a$ the scale factor, and $\Gamma$ is the interaction rate accounting for inverse neutrino decays and majoron decays, given by

$\Gamma=\frac{\Gamma_{\phi}}{2} \frac{m_{\phi}^{2}}{T_{v}^{2}} e^{\frac{\mu_{v}}{T_{v}}} K_{1}\left(\frac{m_{\phi}}{T_{v}}\right)$

where $K_{1}$ is the modified Bessel function of the first kind. For convenience one can approximate $e^{\frac{\mu_{v}}{T_{v}}} \simeq 1$, and $T_{\gamma} / T_{\nu} \simeq$ 1.4 - we have verified that this introduces a negligible error in the final result. In Eq. (5) all derivatives are understood to be with respect to conformal time.

\section{Analysis}

In order to efficiently scan the parameter space of interest, we define an effective interaction $\Gamma_{\text {eff }}$ in terms of the majoron mass and coupling as

$\Gamma_{\text {eff }}=\left(\frac{\lambda}{4 \times 10^{-12}}\right)^{2}\left(\frac{1 \mathrm{keV}}{m_{\phi}}\right)$.

This effective interaction is defined such that for $\Gamma_{\text {eff }} \gtrsim 1$ majorons thermalize in the early Universe. We perform runs with two distinct sets of priors: the first is used to place constraints on majoron models producing strong modifications to the neutrino perturbations, and the second is used to identify parameter space for which the $H_{0}$ tension can be ameliorated. For both sets of runs, we adopt log-flat priors in $\lambda$ or $\Gamma_{\text {eff }}$ and $m_{\phi}$ spanning

$$
\begin{aligned}
\log _{10}(\lambda) & =[-15,-6], \\
\log _{10}\left(m_{\phi} / \mathrm{eV}\right) & =[-2,3],
\end{aligned}
$$

and

$$
\begin{aligned}
\log _{10}\left(\Gamma_{\text {eff }}\right) & =[-4,4], \\
\log _{10}\left(m_{\phi} / \mathrm{eV}\right) & =[-2,2],
\end{aligned}
$$

respectively. In addition to these two parameters, we also allow for the possibility of extra relativistic and noninteracting degrees of freedom. We allow $\Delta N_{\text {eff }}$ to vary linearly between $-2 \leq \Delta N_{\text {eff }} \leq 4$, and treat this additional radiation as free streaming. This additional contribution to $N_{\text {eff }}$ should not be considered ad hoc, but rather a natural expectation of majoron models. For example, should the 
Table 1 Mean (best-fit) values with $\pm 1 \sigma$ errors of the cosmological parameters reconstructed from our combined analysis of Planck2018+BAO+ $\mathrm{SH}_{0}$ ES data in each scenario. For comparison, the best-fit $\chi^{2}$ we find for $\Lambda$ CDM using Planck2018+BAO data only

\begin{tabular}{|c|c|c|c|}
\hline Parameter & $\Lambda \mathrm{CDM}$ & $\Lambda \mathrm{CDM}+\Delta N_{\mathrm{eff}}$ & Majoron $+\Delta N_{\text {eff }}$ \\
\hline$\Delta N_{\text {eff }}$ & - & $0.43(0.358) \pm 0.18$ & $0.52(0.545) \pm 0.19$ \\
\hline$m_{\phi} / \mathrm{eV}$ & - & - & $(0.33)$ \\
\hline$\Gamma_{\text {eff }}$ & - & - & $(8.1)$ \\
\hline $100 \Omega_{b} h^{2}$ & $2.252(2.2563) \pm 0.016$ & $2.270(2.2676) \pm 0.017$ & $2.280(2.2765) \pm 0.02$ \\
\hline$\Omega_{\mathrm{cdm}} h^{2}$ & $0.1176(0.11769) \pm 0.0012$ & $0.125(0.1243) \pm 0.003$ & $0.127(0.1279) \pm 0.004$ \\
\hline $100 \theta_{s}$ & $1.0421(1.04223) \pm 0.0003$ & $1.0411(1.04125) \pm 0.0005$ & $1.0410(1.04102) \pm 0.0005$ \\
\hline $\ln \left(10^{10} A_{s}\right)$ & $3.09(3.1102) \pm 0.03$ & $3.10(3.072) \pm 0.03$ & $3.11(3.116) \pm 0.03$ \\
\hline$n_{s}$ & $0.971(0.9690) \pm 0.004$ & $0.981(0.9780) \pm 0.006$ & $0.990(0.99354) \pm 0.010$ \\
\hline$\tau_{\text {reio }}$ & $0.051(0.0500) \pm 0.008$ & $0.052(0.0537) \pm 0.008$ & $0.052(0.0576) \pm 0.008$ \\
\hline$H_{0}$ & $68.98(69.04) \pm 0.57$ & $71.27(70.60) \pm 1.1$ & $71.92(71.53) \pm 1.2$ \\
\hline$(R-1)_{\min }$ & 0.009 & 0.009 & 0.03 \\
\hline$\chi_{\min }^{2}$ high- $\ell$ & 2341.56 & 2345.39 & 2338.84 \\
\hline$\chi_{\min }^{2}$ lowl & 22.45 & 21.56 & 20.81 \\
\hline$\chi_{\min }^{2}$ lowE & 395.72 & 395.89 & 396.40 \\
\hline$\chi_{\min }^{2}$ lensing & 9.91 & 9.21 & 10.69 \\
\hline$\chi_{\min }^{2} \mathrm{BAO}$ & 4.74 & 4.5 & 4.69 \\
\hline$\chi_{\min }^{2} \mathrm{SH}_{0} \mathrm{ES}$ & 12.34 & 5.82 & 3.10 \\
\hline$\chi_{\min }^{2} \mathrm{CMB}$ & 2769.6 & 2772.1 & 2766.7 \\
\hline$\chi_{\min }^{2}$ TOT & 2786.7 & 2782.4 & 2774.5 \\
\hline$\chi_{\min }^{2}-\left.\chi_{\min }^{2}\right|^{\Lambda \mathrm{CDM}}$ & 0 & -4.3 & -12.2 \\
\hline
\end{tabular}

with $(R-1)_{\min }=0.007$ is: $\chi_{\text {high }-\ell}^{2}=2340.25, \chi_{\text {lowl }}^{2}=22.54$, $\chi_{\text {lowE }}^{2}=395.74, \chi_{\text {lensing }}^{2}=8.92, \chi_{\mathrm{BAO}}^{2}=3.57, \chi_{\mathrm{CMB}}^{2}=2767.45$ reheating temperature be above the mass of right handed neutrinos, a thermal population of majorons produced in the early Universe may come to dominate the energy density of the Universe, producing nearly arbitrarily large contributions to $\Delta N_{\text {eff }}$. Such an effect becomes increasingly important for feeble interactions, such that an effective lower bound can be placed on the the neutrino-majoron interaction - needless to say, however, this bound is inherently dependent on preBBN cosmology. We include in Fig. 1 a line, labeled $\Delta N_{\mathrm{eff}}^{*}$, that identifies parameter space for which the contribution to $\Delta N_{\text {eff }}$ from a primordial population of majorons would be excluded by Planck and measurements of large scale structure. We include a more comprehensive discussion of this effect in the Supplementary Material.

\section{Results and conclusions}

After implementing the above modifications to both the energy density and neutrino-majoron perturbations in CLASS $[107,108]$, we perform an MCMC with Montepython [109,110] using the Planck-2018 TTTEEE+lowlTT+ lowE+lensing likelihood [36], including data on BAOs from the 6DF galaxy survey [111], the MGS galaxy sample of
SDSS [112], and from the CMASS and LOWZ galaxy samples of BOSS DR12 [113], both including and excluding a Gaussian contribution to the likelihood on $H_{0}$ from $\mathrm{SH}_{0} \mathrm{ES}$ [43], taken to have a mean value and standard deviation of 74.0 and $1.4 \mathrm{~km} / \mathrm{s} / \mathrm{Mpc}$. All MCMCs have been run until the largest Gelman-Rubin coefficient was $R-1<0.03$ or better. In Table 1 we outline all relevant cosmological parameters for the analyses of Planck $2018+\mathrm{BAO}+\mathrm{SH}_{0} \mathrm{ES}$ data.

In Fig. 1 we show the $95 \%$ exclusion contours derived in this work, and the $1 \sigma$ contour for parameter space preferred from the fit including the $\mathrm{SH}_{0} \mathrm{ES}$ likelihood. We derive the 95\% CL exclusion contours using only Planck data in order to remain conservative, and note that including e.g. BAO data leads to a minor strengthening of this contour. Interestingly, the results obtained here illustrate that Planck has already begun to significantly probe well-motivated regions of parameter space in which the majoron mass arises from dim-5 Planck scale suppressed operators. If interpreted in terms of the type-I seesaw model, current CMB observations are now probing lepton symmetry breaking scales $\mathcal{O}(100)$ $\mathrm{GeV}$, with future $\mathrm{CMB}$ experiments potentially reaching the level of $\sim 10 \mathrm{TeV}$. Before continuing, we would like to emphasize that the constraints derived in this work are both stringent and robust over wide regions of parameter space. 


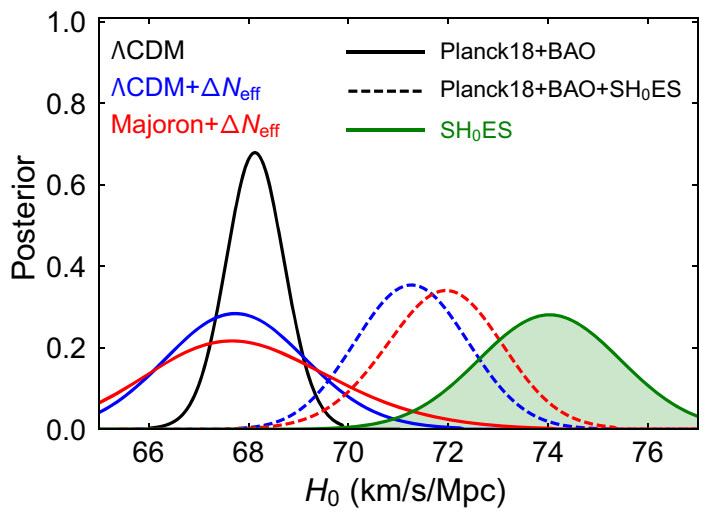

Fig. $2 H_{0}$ posteriors for $\Lambda \mathrm{CDM}$ (black), $\Lambda \mathrm{CDM}+\Delta N_{\text {eff }}$ (blue), and majoron $+\Delta N_{\text {eff }}$ (red), using Planck2018 $+\mathrm{BAO}$ (solid) and including a gaussian likelihood for $\mathrm{SH}_{0} \mathrm{ES}$ (dashed). $\mathrm{SH}_{0} \mathrm{ES}$ posterior shown for comparison in green. See Table 1 for best-fit values and $1 \sigma$ uncertainties. The red solid line roughly corresponds to $H_{0}=68.0 \pm 1.9$ $\mathrm{km} / \mathrm{s} / \mathrm{Mpc}$ and hence is in $2.5 \sigma$ tension with the $\mathrm{SH}_{0} \mathrm{ES}$ measurement

For example, a majoron of $m_{\phi}=1 \mathrm{eV}$ and $\lambda=10^{-11}$ is excluded by more than $5 \sigma$.

In Fig. 2 we show the posterior distributions for $\Lambda \mathrm{CDM}$, $\Lambda \mathrm{CDM}$ with a floating value of $\Delta N_{\text {eff }}$, and the majoron + $\Delta N_{\text {eff }}$, each including and excluding the $\mathrm{SH}_{0} \mathrm{ES}$ likelihood. The $\mathrm{SH}_{0} \mathrm{ES}$ posterior is shown for comparison. Including the majoron broadens the posterior and induces a minimal shift of the central value to large $H_{0}$, an effect which is more visible when the $\mathrm{SH}_{0} \mathrm{ES}$ likelihood is included. While the difference induced by including the majoron is not enormous, the $H_{0}$ tension can be reduced from $4.4 \sigma$ to $2.5 \sigma$ when neutrinomajoron interactions, and an additional contribution to dark radiation, are included.

By performing a MCMC including the $\mathrm{SH}_{0} \mathrm{ES}$ likelihood, we find that a scenario with $\Delta N_{\text {eff }}=0.52 \pm 0.19$, $0.1 \mathrm{eV}<m_{\phi}<1 \mathrm{eV}$, and coupling strengths $\lambda \sim\left(10^{-14}-\right.$ $\left.10^{-13}\right)\left(\mathrm{eV} / m_{\phi}\right)$ - as highlighted in red in Fig. 1 - would render a posterior for $H_{0}$ of $71.9 \pm 1.2 \mathrm{~km} / \mathrm{s} / \mathrm{Mpc}$ and an overall improvement of $\Delta \chi^{2} \simeq-12.2$ with respect to $\Lambda \mathrm{CDM}$. We remind the reader here that, because of the residual $2.5 \sigma$ tension, it may not be entirely meaningful to combine the partially discrepant datasets, and thus care should be given in the interpretation of this region. Notice that the improvement in the $\chi^{2}$ does not exclusively arise from the shift in $H_{0}$; this can be seen from the fact that the contribution of the CMB likelihood in the Majoron $+\Delta N_{\text {eff }}$ is less than that of $\Lambda C D M$. Interestingly, this region of parameter space corresponds to lepton number symmetry breaking scales in the type-I seesaw near the electroweak scale. Furthermore, it is worth emphasizing that unlike the strongly interacting neutrino solution proposed in [30] (defined by a 2-to-2 neutrino contact interaction), the solution proposed here is robust to the inclusion of polarization data, is phenomenologically viable, and is theoretically motivated.
An important comment on the consistency of this type of solution is necessary. If the contribution to $N_{\text {eff }}$ is of primordial origin, then successful BBN excludes values of $\Delta N_{\text {eff }} \gtrsim 0.4$ at $T \sim \operatorname{MeV}[87,114,115]$. In addition, including a floating value of $\Delta N_{\text {eff }}$ in the CMB analysis can induce a shift in the preferred value of $\Omega_{b} h^{2}$, which is also constrained by BBN. In the Supplementary Material, we address the extent to which the parameter space in the $\Delta N_{\text {eff }}-\Omega_{b} h^{2}$ plane preferred by the CMB fit is compatible with expectations of BBN.

Evidence for the existence of the majoron, arising from the spontaneous breaking of global lepton number, would provide a strong clue to the origin of the neutrino masses. In this work we have looked at the extent to which $\mathrm{CMB}$ measurements have probed the existence of such a particle through its impact on the expansion history of the Universe and its interactions with neutrinos. We show that there exists a broad range of well-motivated parameter space that is now excluded using Planck2018 measurements of the CMB power spectrum. Furthermore, we identify a region in which the majoron interactions help ameliorate the outstanding $H_{0}$ tension to a level that is beyond what is simply accomplished by including $\Delta N_{\text {eff. }}$. If confirmed, the $H_{0}$ tension could be providing the first insight into the origin of the small neutrino masses.

Acknowledgements The authors thank Isabel Oldengott and Olga Mena for useful discussions. ME is supported by the European Research Council under the European Union's Horizon 2020 program (ERC Grant Agreement No. 648680 DARKHORIZONS). SJW would like to thank ME and the TPPC group at King's College, as well as the Fermilab theory group, for the hospitality during the extended stays that lead to the completion of this work. SJW acknowledges support under Spanish Grants FPA2014-57816-P and FPA2017-85985-P of the MINECO and PROMETEO II/2014/050 of the Generalitat Valenciana, and from the European Union's Horizon 2020 research and innovation program under the Marie Skłodowska-Curie Grant Agreements Nos. 690575 and 674896.

Data Availability Statement This manuscript has no associated data or the data will not be deposited. [Authors' comment: This paper is based on Planck legacy data presented in Refs. [35,36].]

Open Access This article is licensed under a Creative Commons Attribution 4.0 International License, which permits use, sharing, adaptation, distribution and reproduction in any medium or format, as long as you give appropriate credit to the original author(s) and the source, provide a link to the Creative Commons licence, and indicate if changes were made. The images or other third party material in this article are included in the article's Creative Commons licence, unless indicated otherwise in a credit line to the material. If material is not included in the article's Creative Commons licence and your intended use is not permitted by statutory regulation or exceeds the permitted use, you will need to obtain permission directly from the copyright holder. To view a copy of this licence, visit http://creativecomm ons.org/licenses/by/4.0/.

Funded by $\mathrm{SCOAP}^{3}$. 


\section{References}

1. R.N. Mohapatra, G. Senjanovic, Neutrino masses and mixings in gauge models with spontaneous parity violation. Phys. Rev. D 23, 165 (1981). https://doi.org/10.1103/PhysRevD.23.165

2. K.S. Babu, Model of 'Calculable' Majorana neutrino masses. Phys. Lett. B 203, 132 (1988). https://doi.org/10.1016/ 0370-2693(88)91584-5

3. A. Pilaftsis, Radiatively induced neutrino masses and large Higgs neutrino couplings in the standard model with Majorana fields. Z. Phys. C 55, 275 (1992). https://doi.org/10.1007/BF01482590. arXiv:hep-ph/9901206

4. S.F. King, Neutrino mass models. Rept. Progr. Phys. 67, 107 (2004). https://doi.org/10.1088/0034-4885/67/2/R01. arXiv:hep-ph/0310204

5. G. Altarelli, F. Feruglio, Models of neutrino masses and mixings. New J. Phys. 6, 106 (2004). https://doi.org/10.1088/1367-2630/ 6/1/106. arXiv:hep-ph/0405048

6. R.N. Mohapatra et al., Theory of neutrinos: a white paper. Rept. Progr. Phys. 70, 1757 (2007). https://doi.org/10.1088/0034-4885/ 70/11/R02. arXiv:hep-ph/0510213

7. P. Minkowski, $\mu \rightarrow e \gamma$ at a rate of one out of $10^{9}$ Muon decays? Phys. Lett. B 67, 421 (1977). https://doi.org/10.1016/ 0370-2693(77)90435-X

8. R.N. Mohapatra, G. Senjanovic, Neutrino mass and spontaneous parity nonconservation. Phys. Rev. Lett. 44, 912 (1980). https:// doi.org/10.1103/PhysRevLett.44.912

9. M. Gell-Mann, P. Ramond, R. Slansky, Complex spinors and unified theories. Conf. Proc. C 790927, 315 (1979). arXiv:1306.4669

10. T. Yanagida, Horizontal symmetry and masses of neutrinos. Progr. Theor. Phys. 64, 1103 (1980). https://doi.org/10.1143/PTP.64. 1103

11. J. Schechter, J.W.F. Valle, Neutrino masses in $\mathrm{SU}(2) \mathrm{x} U(1)$ theoriesD. Phys. Rev. D 22, 2227 (1980). https://doi.org/10.1103/ PhysRevD.22.2227

12. Y. Chikashige, R.N. Mohapatra, R.D. Peccei, Are there real goldstone bosons associated with broken lepton number? Phys. Lett. B 98, 265 (1981). https://doi.org/10.1016/0370-2693(81)90011-3

13. G.B. Gelmini, M. Roncadelli, Left-handed neutrino mass scale and spontaneously broken lepton number. Phys. Lett. B 99, 411 (1981). https://doi.org/10.1016/0370-2693(81)90559-1

14. H.M. Georgi, S.L. Glashow, S. Nussinov, Unconventional model of neutrino masses. Nucl. Phys. B 193, 297 (1981). https://doi. org/10.1016/0550-3213(81)90336-9

15. J. Schechter, J.W.F. Valle, Neutrino decay and spontaneous violation of lepton number. Phys. Rev. D 25, 774 (1982). https://doi. org/10.1103/PhysRevD.25.774

16. S. Bashinsky, U. Seljak, Neutrino perturbations in CMB anisotropy and matter clustering. Phys. Rev. D 69, 083002 (2004). $\quad$ https://doi.org/10.1103/PhysRevD.69.083002. arXiv:astro-ph/0310198

17. Z. Chacko, L.J. Hall, T. Okui, S.J. Oliver, CMB signals of neutrino mass generation. Phys. Rev. D 70, 085008 (2004). https://doi.org/ 10.1103/PhysRevD.70.085008. arXiv:hep-ph/0312267

18. S. Hannestad, Structure formation with strongly interacting neutrinos-implications for the cosmological neutrino mass bound. JCAP 0502, 011 (2005). https://doi.org/10.1088/ 1475-7516/2005/02/011. arXiv:astro-ph/0411475

19. S. Hannestad, G. Raffelt, Constraining invisible neutrino decays with the cosmic microwave background. Phys. Rev. D 72, 103514 (2005). https://doi.org/10.1103/PhysRevD.72.103514. arXiv: hep-ph/0509278

20. N.F. Bell, E. Pierpaoli, K. Sigurdson, Cosmological signatures of interacting neutrinos. Phys. Rev. D 73, 063523 (2006). https://doi. org/10.1103/PhysRevD.73.063523. arXiv:astro-ph/0511410
21. A. Friedland, K. M. Zurek, S. Bashinsky, Constraining models of neutrino mass and neutrino interactions with the planck satellite. arXiv: 0704.3271

22. C. Brust, Y. Cui, K. Sigurdson, Cosmological constraints on interacting light particles. JCAP 1708, 020 (2017). https://doi.org/10. 1088/1475-7516/2017/08/020. arXiv: 1703.10732

23. L.A. Anchordoqui, H. Goldberg, G. Steigman, Right-handed neutrinos as the dark radiation: status and forecasts for the LHC. Phys. Lett. B 718, 1162 (2013). https://doi.org/10.1016/j.physletb.2012. 12.019. arXiv: 1211.0186

24. R. Diamanti, E. Giusarma, O. Mena, M. Archidiacono, A. Melchiorri, Dark radiation and interacting scenarios. Phys. Rev. D 87, 063509 (2013). https://doi.org/10.1103/PhysRevD.87. 063509. arXiv: 1212.6007

25. M. Archidiacono, S. Hannestad, Updated constraints on nonstandard neutrino interactions from Planck. JCAP 1407, 046 (2014). https://doi.org/10.1088/1475-7516/2014/07/046. arXiv: 1311.3873

26. I.M. Oldengott, C. Rampf, Y.Y.Y. Wong, Boltzmann hierarchy for interacting neutrinos I: formalism. JCAP 1504, 016 (2015). https://doi.org/10.1088/1475-7516/2015/04/016. arXiv: 1409.1577

27. I.M. Oldengott, T. Tram, C. Rampf, Y.Y.Y. Wong, Interacting neutrinos in cosmology: exact description and constraints. JCAP 1711, 027 (2017). https://doi.org/10.1088/1475-7516/2017/11/ 027. arXiv: 1706.02123

28. F. Forastieri, M. Lattanzi, P. Natoli, Constraints on secret neutrino interactions after Planck. JCAP 1507, 014 (2015). https://doi.org/ 10.1088/1475-7516/2015/07/014. arXiv:1504.04999

29. L. Lancaster, F.-Y. Cyr-Racine, L. Knox, Z. Pan, A tale of two modes: Neutrino free-streaming in the early universe. JCAP 1707, 033 (2017). https://doi.org/10.1088/1475-7516/2017/07/ 033. arXiv: 1704.06657

30. C.D. Kreisch, F.-Y. Cyr-Racine, O. Doré, The neutrino puzzle: anomalies, interactions, and cosmological tensions. arXiv: 1902.00534

31. M. Park, C. D. Kreisch, J. Dunkley, B. Hadzhiyska, F.-Y. CyrRacine, $\Lambda$ CDM or self-interacting neutrinos? How CMB data can tell the two models apart. arXiv: 1904.02625

32. F. Forastieri, M. Lattanzi, P. Natoli, Cosmological constraints on neutrino self-interactions with a light mediator. arXiv:904.07810

33. G. Barenboim, P.B. Denton, I.M. Oldengott, Constraints on inflation with an extended neutrino sector. Phys. Rev. D 99, 083515 (2019). https://doi.org/10.1103/PhysRevD.99.083515. arXiv: 1903.02036

34. M. Escudero, M. Fairbairn, Cosmological constraints on invisible neutrino decays revisited. Phys. Rev. D 100, 103531 (2019). https://doi.org/10.1103/PhysRevD.100.103531. arXiv: 1907.05425

35. PLANCK collaboration, N. Aghanim et al., Planck 2018 results. VI. Cosmological parameters. arXiv:1807.06209

36. PlANCK Collaboration, N. Aghanim et al., Planck 2018 results. V. CMB power spectra and likelihoods. arXiv:1907.12875

37. SimOns OBSERVATORY Collaboration, J. Aguirre et al., The simons observatory: science goals and forecasts, JCAP 1902, 056 (2019). https://doi.org/10.1088/1475-7516/2019/02/ 056. arXiv: 1808.07445

38. K. Abazajian et al., CMB-S4 Science Case, Reference design, and project plan. arXiv: 1907.04473

39. G.E. Addison, D.J. Watts, C.L. Bennett, M. Halpern, G. Hinshaw, J.L. Weiland, Elucidating $\Lambda$ CDM: impact of baryon acoustic oscillation measurements on the hubble constant discrepancy. Astrophys. J. 853, 119 (2018). https://doi.org/10.3847/ 1538-4357/aaa1ed. arXiv:1707.06547 
40. A. Cuceu, J. Farr, P. Lemos, A. Font-Ribera, Baryon acoustic oscillations and the hubble constant: past, present and future. arXiv: 1906.11628

41. N. Schöneberg, J. Lesgourgues, D.C. Hooper, The BAO + BBN take on the Hubble tension. arXiv:1907.11594

42. A.G. Riess et al., A $2.4 \%$ determination of the local value ofthe hubble constant. Astrophys. J. 826, 56 (2016). https://doi.org/10. 3847/0004-637X/826/1/56. arXiv:1604.01424

43. A.G. Riess, S. Casertano, W. Yuan, L.M. Macri, D. Scolnic, Large magellanic cloud cepheid standards provide a $1 \%$ foundation for the determination of the hubble constant and stronger evidence for physics beyond $\Lambda$ CDM. Astrophys. J. 876, 85 (2019). https:// doi.org/10.3847/1538-4357/ab1422. arXiv:1903.07603

44. S. Dhawan, S.W. Jha, B. Leibundgut, Measuring the hubble constant with type ia supernovae as near-infrared standard candles. Astron. Astrophys. A 609, 72 (2018). https://doi.org/10.1051/ 0004-6361/201731501. arXiv: 1707.00715

45. CSP Collaboration, C. R. Burns et al., The carnegie supernova project: absolute calibration and the hubble constant. Astrophys. J. 869, 56 (2018). https://doi.org/10.3847/1538-4357/aae51c. arXiv: 1809.06381

46. W.L. Freedman et al., The Carnegie-Chicago hubble program. VIII. An independent determination of the hubble constant based on the tip of the red giant branch. arXiv:1907.05922

47. W. Yuan, A. G. Riess, L. M. Macri, S. Casertano, D. Scolnic, Consistent calibration of the tip of the red giant branch in the large magellanic cloud on the hubble space telescope photometric system and implications for the determination of the hubble constant. arXiv: 1908.00993

48. V. Bonvin et al., HOLiCOW-V. New COSMOGRAIL time delays of HE 0435-1223: $H_{0}$ to 3.8 per cent precision from strong lensing in a flat $\Lambda \mathrm{CDM}$ model. Mon. Not. R. Astron. Soc. 465, 4914 (2017). https://doi.org/10.1093/mnras/stw3006. arXiv: 1607.01790

49. S. Birrer et al., HOLiCOW - IX. Cosmographic analysis of the doubly imaged quasar SDSS $1206+4332$ and a new measurement of the Hubble constant. Mon. Not. R. Astron. Soc. 484, 4726 (2019). https://doi.org/10.1093/mnras/stz200. arXiv:1809.01274

50. C. E. Rusu et al., HOLiCOW XII. Lens mass model of WFI20334723 and blind measurement of its time-delay distance and $H_{0}$. arXiv: 1905.09338

51. G.C.F. Chen et al., A SHARP view of HOLiCOW: $H_{0}$ from three time-delay gravitational lens systems with adaptive optics imaging. arXiv: 1907.02533

52. L. Verde, T. Treu, A. G. Riess, Tensions between the early and the late universe (2019). arXiv:1907.10625

53. K.C. Wong et al., HOLiCOW XIII. A $2.4 \%$ measurement of $H_{0}$ from lensed quasars: $5.3 \sigma$ tension between early and lateUniverse probes. arXiv: 1907.04869

54. J.L. Bernal, L. Verde, A.G. Riess, The trouble with $H_{0}$. JCAP 1610, 019 (2016). https://doi.org/10.1088/1475-7516/2016/10/ 019. arXiv: 1607.05617

55. E. Mörtsell, S. Dhawan, Does the Hubble constant tension call for new physics? JCAP 1809, 025 (2018). https://doi.org/10.1088/ 1475-7516/2018/09/025. arXiv:1801.07260

56. F. D'Eramo, R.Z. Ferreira, A. Notari, J.L. Bernal, Hot axions and the $H_{0}$ tension. JCAP 1811, 014 (2018). https://doi.org/10.1088/ 1475-7516/2018/11/014. arXiv:1808.07430

57. M. Escudero, D. Hooper, G. Krnjaic, M. Pierre, Cosmology with a very light $\mathrm{L}_{\mu}-\mathrm{L}_{\tau}$ Gauge boson. JHEP 03, 071 (2019). https:// doi.org/10.1007/JHEP03(2019)071. arXiv:1901.02010

58. G.B. Gelmini, A. Kusenko, V. Takhistov, Hints of sterile neutrinos in recent measurements of the hubble parameter. arXiv: 1906.10136

59. M. Archidiacono, S. Gariazzo, C. Giunti, S. Hannestad, R. Hansen, M. Laveder et al., Pseudoscalar-sterile neutrino interac- tions: reconciling the cosmos with neutrino oscillations. JCAP 1608, 067 (2016). https://doi.org/10.1088/1475-7516/2016/08/ 067. arXiv: 1606.07673

60. E. Di Valentino, C. Bœehm, E. Hivon, F.R. Bouchet, Reducing the $H_{0}$ and $\sigma_{8}$ tensions with dark matter-neutrino interactions. Phys. Rev. D 97, 043513 (2018). https://doi.org/10.1103/PhysRevD.97. 043513. arXiv: 1710.02559

61. S. Ghosh, R. Khatri, T. S. Roy, Dark Neutrino interactions phase out the Hubble tension. arXiv:1908.09843

62. E. Di Valentino, A. Melchiorri, J. Silk, Reconciling Planck with the local value of $H_{0}$ in extended parameter space. Phys. Lett. B 761, 242 (2016). https://doi.org/10.1016/j.physletb.2016.08.043. arXiv: 1606.00634

63. Q.-G. Huang, K. Wang, How the dark energy can reconcile Planck with local determination of the Hubble constant. Eur. Phys. J. C 76, 506 (2016). https://doi.org/10.1140/epjc/ s10052-016-4352-x. arXiv:1606.05965

64. T. Karwal, M. Kamionkowski, Dark energy at early times, the Hubble parameter, and the string axiverse. Phys. Rev. D 94, 103523 (2016). https://doi.org/10.1103/PhysRevD.94.103523. arXiv: 1608.01309

65. P. Ko, Y. Tang, Light dark photon and fermionic dark radiation for the Hubble constant and the structure formation. Phys. Lett. B 762, 462 (2016). https://doi.org/10.1016/j.physletb.2016.10.001. arXiv: 1608.01083

66. E. Di Valentino, A. Melchiorri, O. Mena, Can interacting dark energy solve the $H_{0}$ tension? Phys. Rev. D 96, 043503 (2017). https://doi.org/10.1103/PhysRevD.96.043503. arXiv: 1704.08342

67. E. Di Valentino, E.V. Linder, A. Melchiorri, Vacuum phase transition solves the $H_{0}$ tension. Phys. Rev. D 97, 043528 (2018). https://doi.org/10.1103/PhysRevD.97.043528. arXiv: 1710.02153

68. V. Poulin, T.L. Smith, T. Karwal, M. Kamionkowski, Early dark energy can resolve the hubble tension. Phys. Rev. Lett. 122, 221301 (2019). https://doi.org/10.1103/PhysRevLett.122. 221301. arXiv: 1811.04083

69. E. Di Valentino, R. Z. Ferreira, L. Visinelli, U. Danielsson, Late time transitions in the quintessence field and the $H_{0}$ tension. arXiv: 1906.11255

70. P. Agrawal, F.-Y. Cyr-Racine, D. Pinner, L. Randall, Rock 'n' roll solutions to the hubble tension. arXiv: 1904.01016

71. S. Alexander, E. McDonough, Axion-Dilaton destabilization and the hubble tension. arXiv:1904.08912

72. M.-X. Lin, G. Benevento, W. Hu, M. Raveri, Acoustic dark energy: potential conversion of the hubble tension. arXiv: 1905.12618

73. P. Agrawal, G. Obied, C. Vafa, $H_{0}$ Tension, swampland conjectures and the epoch of fading dark matter. arXiv:1906.08261

74. E. Di Valentino, A. Melchiorri, O. Mena, S. Vagnozzi, Interacting dark energy after the latest Planck, DES, and $H_{0}$ measurements: an excellent solution to the $H_{0}$ and cosmic shear tensions. arXiv: 1908.04281

75. T. L. Smith, V. Poulin, M. A. Amin, Oscillating scalar fields and the Hubble tension: a resolution with novel signatures. arXiv: 1908.06995

76. T. Bringmann, F. Kahlhoefer, K. Schmidt-Hoberg, P. Walia, Converting nonrelativistic dark matter to radiation. Phys. Rev. D 98, 023543 (2018). https://doi.org/10.1103/PhysRevD.98. 023543. arXiv: 1803.03644

77. K. L. Pandey, T. Karwal, S. Das, Alleviating the $H_{0}$ and $\sigma_{8}$ anomalies with a decaying dark matter model. arXiv:1902.10636

78. M. Raveri, W. Hu, T. Hoffman, L.-T. Wang, Partially acoustic dark matter cosmology and cosmological constraints. Phys. Rev. D 96, 103501 (2017). https://doi.org/10.1103/PhysRevD.96. 103501. arXiv: 1709.04877 
79. W. Yang, S. Pan, S. Vagnozzi, E. Di Valentino, D. F. Mota, S. Capozziello, Dawn of the dark: unified dark sectors and the EDGES Cosmic Dawn 21-cm signal. arXiv:1907.05344

80. J. Renk, M. Zumalacárregui, F. Montanari, A. Barreira, Galileon gravity in light of ISW, CMB, BAO and $\mathrm{H}_{0}$ data. JCAP 1710, 020 (2017). https://doi.org/10.1088/1475-7516/2017/10/ 020. arXiv: 1707.02263

81. N. Khosravi, S. Baghram, N. Afshordi, N. Altamirano, $H_{0}$ tension as a hint for a transition in gravitational theory. Phys. Rev. D 99, 103526 (2019). https://doi.org/10.1103/PhysRevD.99. 103526. arXiv: 1710.09366

82. M.-X. Lin, M. Raveri, W. Hu, Phenomenology of modified gravity at recombination. Phys. Rev. D 99, 043514 (2019). https://doi.org/ 10.1103/PhysRevD.99.043514. arXiv:1810.02333

83. M. Martinelli, I. Tutusaus, CMB tensions with low-redshift $H_{0}$ and $S_{8}$ measurements: impact of a redshift-dependent type-Ia supernovae intrinsic luminosity. arXiv:1906.09189

84. L. Knox, M. Millea, The Hubble Hunter's Guide. arXiv: 1908.03663

85. S. Vagnozzi, New physics in light of the $H_{0}$ tension: an alternative view. arXiv:1907.07569

86. N. Blinov, K.J. Kelly, G.Z. Krnjaic, S.D. McDermott, Constraining the self-interacting neutrino interpretation of the hubble tension. arXiv: 1905.02727

87. C. Pitrou, A. Coc, J.-P. Uzan, E. Vangioni, Precision big bang nucleosynthesis with improved Helium-4 predictions. Phys. Rept. 754, 1 (2018). https://doi.org/10.1016/j.physrep.2018.04. 005. arXiv: 1801.08023

88. ParticleDataGroup collaboration, M. Tanabashi et al., Review of particle physics. Phys. Rev. D 98, 030001 (2018). https://doi. org/10.1103/PhysRevD.98.030001

89. KAMLAND- ZEN collaboration, A. Gando et al., Limits on Majoron-emitting double-beta decays of Xe-136 in the KamLAND-Zen experiment, Phys. Rev. C 86, 021601 (2012). https://doi.org/10.1103/PhysRevC.86.021601. arXiv:1205.6372

90. M. Kachelriess, R. Tomas, J.W.F. Valle, Supernova bounds on Majoron emitting decays of light neutrinos. Phys. Rev. D 62, 023004 (2000). https://doi.org/10.1103/PhysRevD.62.023004. arXiv:hep-ph/0001039

91. Y. Farzan, Bounds on the coupling of the Majoron to light neutrinos from supernova cooling. Phys. Rev. D 67, 073015 (2003). https://doi.org/10.1103/PhysRevD.67.073015. arXiv:hep-ph/0211375

92. T. Banks, N. Seiberg, Symmetries and strings in field theory and gravity. Phys. Rev. D 83, 084019 (2011). https://doi.org/10.1103/ PhysRevD.83.084019. arXiv:1011.5120

93. E. Witten, Symmetry and emergence. Nat. Phys. 14, 116 (2018). https://doi.org/10.1038/nphys4348. arXiv:1710.01791

94. I.Z. Rothstein, K.S. Babu, D. Seckel, Planck scale symmetry breaking and majoron physics. Nucl. Phys. B 403, 725 (1993). https://doi.org/10.1016/0550-3213(93)90368-Y. arXiv:hep-ph/9301213

95. E.K. Akhmedov, Z.G. Berezhiani, R.N. Mohapatra, G. Senjanovic, Planck scale effects on the majoron. Phys. Lett. B 299, 90 (1993). https://doi.org/10.1016/0370-2693(93)90887-N. arXiv:hep-ph/9209285

96. R. Kallosh, A.D. Linde, D.A. Linde, L. Susskind, Gravity and global symmetries. Phys. Rev. D 52, 912 (1995). https://doi.org/ 10.1103/PhysRevD.52.912. arXiv:hep-th/9502069

97. S. Chang, K. Choi, Constraints from nucleosynthesis and SN1987A on majoron emitting double beta decay. Phys. Rev. D 49, 12 (1994). https://doi.org/10.1103/PhysRevD.49.R12. arXiv:hep-ph/9303243

98. M. Escudero, Precision Early Universe Thermodynamics made simple: $N_{\text {eff }}$ and Neutrino Decoupling in the Standard Model and beyond. arXiv:2001.04466 [hep-ph]
99. M. Escudero, Neutrino decoupling beyond the standard model: CMB constraints on the Dark Matter mass with a fast and precise

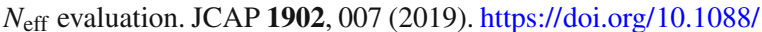
1475-7516/2019/02/007. arXiv:1812.05605

100. CMB- S4 Collaboration, K.N. Abazajian et al., CMB-S4 Science Book, First Edition. arXiv: 1610.02743

101. S. Vagnozzi, E. Giusarma, O. Mena, K. Freese, M. Gerbino, S. Ho et al., Unveiling $v$ secrets with cosmological data: neutrino masses and mass hierarchy. Phys. Rev. D 96, 123503 (2017). https://doi. org/10.1103/PhysRevD.96.123503. arXiv: 1701.08172

102. A. Loureiro et al., On the upper bound of neutrino masses from combined cosmological observations and particle physics experiments. Phys. Rev. Lett. 123, 081301 (2019). https://doi.org/10. 1103/PhysRevLett.123.081301. arXiv: 1811.02578

103. S. Roy Choudhury, S. Hannestad, Updated results on neutrino mass and mass hierarchy from cosmology with Planck 2018 likelihoods. arXiv: 1907.12598

104. S. Vagnozzi, Cosmological searches for the neutrino mass scale and mass ordering, arXiv:1907.08010

105. S. Hannestad, R.J. Scherrer, Selfinteracting warm dark matter. Phys. Rev. D 62, 043522 (2000). https://doi.org/10.1103/ PhysRevD.62.043522. arXiv:astro-ph/0003046

106. C.-P. Ma, E. Bertschinger, Cosmological perturbation theory in the synchronous and conformal Newtonian gauges. Astrophys. J. 455, 7 (1995). https://doi.org/10.1086/176550. arXiv:astro-ph/9506072

107. D. Blas, J. Lesgourgues, T. Tram, The Cosmic linear anisotropy solving system (CLASS) II: approximation schemes. JCAP 1107, 034 (2011). https://doi.org/10.1088/1475-7516/2011/07/ 034. arXiv: 1104.2933

108. J. Lesgourgues, The cosmic linear anisotropy solving system (CLASS) I: overview. arXiv:1104.2932

109. T. Brinckmann, J. Lesgourgues, MontePython 3: boosted MCMC sampler and other features. arXiv: 1804.07261

110. B. Audren, J. Lesgourgues, K. Benabed, S. Prunet, Conservative constraints on early cosmology: an illustration of the Monte Python cosmological parameter inference code. JCAP 1302, 001 (2013). https://doi.org/10.1088/1475-7516/2013/02/ 001. arXiv: 1210.7183

111. F. Beutler, C. Blake, M. Colless, D.H. Jones, L. Staveley-Smith, L. Campbell et al., The 6dF Galaxy survey: baryon acoustic oscillations and the local hubble constant. Mon. Not. R. Astron. Soc. 416, 3017 (2011). https://doi.org/10.1111/j.1365-2966.2011.19250.x. arXiv: 1106.3366

112. A.J. Ross, L. Samushia, C. Howlett, W.J. Percival, A. Burden, M. Manera, The clustering of the SDSS DR7 main Galaxy sample? I. A 4 per cent distance measure at $z=0.15$. Mon. Not. R. Astron. Soc. 449, 835 (2015). https://doi.org/10.1093/mnras/stv154

113. BOSS Collaboration, S. Alam et al., The clustering of galaxies in the completed SDSS-III Baryon oscillation spectroscopic survey: cosmological analysis of the DR12 galaxy sample. Monthly. Not. R. Astron. Soc. 470, 2617 (2017). https://doi.org/10.1093/mnras/ stx721. arXiv: 1607.03155

114. R.H. Cyburt, B.D. Fields, K.A. Olive, T.-H. Yeh, Big Bang Nucleosynthesis: 2015. Rev. Mod. Phys. 88, 015004 (2016). https://doi. org/10.1103/RevModPhys.88.015004. arXiv:1505.01076

115. A. Berlin, N. Blinov, S.W. Li, Dark sector equilibration during nucleosynthesis. Phys. Rev. D 100, 015038 (2019). https://doi. org/10.1103/PhysRevD.100.015038. arXiv:1904.04256

116. P.F. de Salas, S. Pastor, Relic neutrino decoupling with flavour oscillations revisited. JCAP 1607, 051 (2016). https://doi.org/10. 1088/1475-7516/2016/07/051. arXiv:1606.06986

117. G. Mangano, G. Miele, S. Pastor, T. Pinto, O. Pisanti, P.D. Serpico, Relic neutrino decoupling including flavor oscillations. Nucl. Phys. B 729, 221 (2005). https://doi.org/10.1016/j.nuclphysb. 2005.09.041. arXiv:hep-ph/0506164 
118. M. Pospelov, J. Pradler, Big bang nucleosynthesis as a probe of new physics. Ann. Rev. Nucl. Part. Sci. 60, 539 (2010). https:// doi.org/10.1146/annurev.nucl.012809.104521. arXiv:1011.1054

119. F. Iocco, G. Mangano, G. Miele, O. Pisanti, P.D. Serpico, Primordial nucleosynthesis: from precision cosmology to fundamental physics. Phys. Rept. 472, 1 (2009). https://doi.org/10.1016/j. physrep.2009.02.002. arXiv:0809.0631

120. S. Sarkar, Big bang nucleosynthesis and physics beyond the standard model. Rept. Progr. Phys. 59, 1493 (1996). https://doi.org/ 10.1088/0034-4885/59/12/001. arXiv:hep-ph/9602260

121. M.C. Gonzalez-Garcia, A. Santamaria, J.W.F. Valle, Isosinglet Neutral heavy lepton production in $Z$ decays and neutrino mass. Nucl. Phys. B 342, 108 (1990). https://doi.org/10.1016/ 0550-3213(90)90573-V
122. D. Besak, D. Bodeker, Thermal production of ultrarelativistic right-handed neutrinos: complete leading-order results. JCAP 1203, 029 (2012). https://doi.org/10.1088/1475-7516/2012/03/ 029. arXiv: 1202.1288

123. B. Garbrecht, F. Glowna, P. Schwaller, Scattering rates for leptogenesis: damping of lepton flavour coherence and production of singlet neutrinos. Nucl. Phys. B 877, 1 (2013). https://doi.org/10. 1016/j.nuclphysb.2013.08.020. arXiv:1303.5498

124. I. Ghisoiu, M. Laine, Right-handed neutrino production rate at $T>160$ GeV. JCAP 1412, 032 (2014). https://doi.org/10.1088/ 1475-7516/2014/12/032. arXiv:1411.1765 